\title{
Multidisciplinary treatment plan for multiple dental areneses associated with hypohidrotic ectodermal dysplasias
}

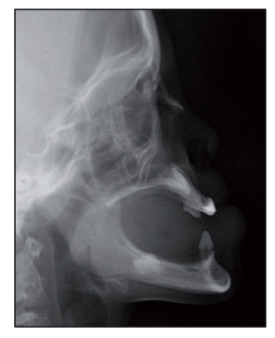

\author{
François CLAUSS, Rémy MATHIS, Frédéric OBRY, \\ Quentin KAMM, Fabienne PERRIN-SCHMITT, \\ Marie-Cécile MANIÈRE
}

\begin{abstract}
Hypohidrotic ectodermal dysplasias (HED) are a heterogeneous and complex group of syndromes characterized by a dental craniofacial phenotype associated with severe oligodontia, maxillary hypoplasia with broad face and most notably facial concavity. Cephalometric analyses show insufficient maxillary sagittal growth, a protruded mandible, reduced facial and ramus heights, as well as basicranial changes. Early diagnosis and care from a multidisciplinary team are essential. In the primary dentition, initial prosthetic treatment is recommended and may possibly be combined with interceptive orthodontic treatment. In the mixed dentition, treatment of a transverse maxillary deficiency consists in installing a removable expander or a quadhelix in the case of moderate phenotypes where there is adequate anchorage support. To achieve mandibular anterior repositioning of the maxilla the patient must wear a facial mask. In the permanent dentition, the important steps are correlated with the pre-implant and pre-prosthetic orthodontic adjustments, as well as the presurgical orthodontic preparation that precedes subsequent single or double jaw orthognathic surgery. Temporary skeletal anchorage with mini-screws or plates can be used in cases of insufficient anchorage.
\end{abstract}

KEY WORDS

Hypohidrotic ectodermal dysplasia

Craniofacial phenotype Oligodontia 
INTRODUCTION

Dental developmental abnormalities, whether isolated or syndromic, may be categorized as abnormalities in number, morphology, size or structure. These abnormalities can occur as an isolated phenotype or as part of a syndrome with multiple deformities, as in the case of hypohidrotic ectodermal dysplasia (HED), associated with clinical scenarios such as oligodontia $^{9}$. The dento-cranio-facial phenotype of HED combining oligodontia and dysmorphic features requires an early and coordinated therapeutic plan managed by a multidisciplinary team ${ }^{2,9,25}$.

\section{1 - PRESENTATION OF CLINICAL AND MOLECULAR ASPECTS OF HYPOHIDROTIC ECTODERMAL DYSPLASIAS}

a) Abnormalities of the various ectodermal structures associated with HED are responsible for the many clinical manifestations of the general phenotype and dentofacial complex ${ }^{8,9}$. From a dermatological standpoint, eczematous lesions, hypotrichosis and agenesis or dystrophia of the sweat glands, cause problems with thermoregulation and have been observed in the various forms of HED ${ }^{26}$. Systemically, the different exocrine glands of the body are also dystrophic, and this is, in particular, the cause of inflammation of the respiratory and digestive tracts. The clinical triad - oligodontia-hypotrichosis-hypofunction of the sweat glands is characteristic of the most prevalent form, X linked HED.

Dominant and recessive autosomal forms are quite similar to $X$ linked HED both globally and from a dento- cranio-facial perspective ${ }^{9}$. Therefore, there are numerous orofacial clinical manifestations involving esthetic repercussions such as oligodontia, collapsed vertical dimension, characteristic dysmorphism and in particular hypotrichosis.

b) On a molecular level, the most prevalent forms of HED are $X$ linked $H E D$, caused by mutations of the EDA gene and autosomal HEDs that are linked to mutations of the EDAREDARADD genes ${ }^{6}$. These mutations are involved with the various components of the signaling pathway of $E D A-N F-K B$. In fact, the EDA gene codes for a TNF-like molecule called ectodysplasin (EDA), is a morphogenic factor implicated in the early phases of ectodermal development, as well as in dental morphogenesis.

\section{2 - DENTAL PHENOTYPIC, DENTOALVEOLAR AND CRANIOFACIAL FEATURES ASSOCIATED WITH HED}

a) Oligodontia associated with HED is usually severe, in the temporary as well as in the permanent dentition and has multiple morphologic and 


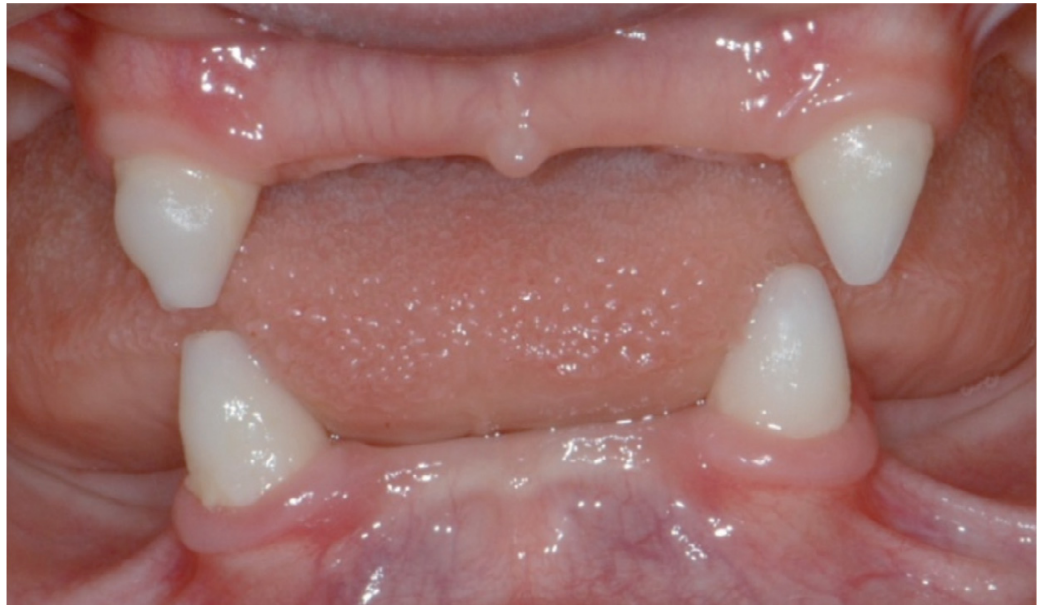

Figure 1

Clinical phenotype associated with hypohidrotic ectodermal dysplasia in the $x$-linked form: oligodontia, cone-shaped canines.

functional consequences ${ }^{9}$. Subsequently, gaps, alveolar bone dysplasia, and decreased lower facial height with incisor overbite are some of the examples of the clinical features directly linked to this dental phenotype (Fig 1-2).

On average, there are 8 congenitally missing teeth in the temporary dentition, and an average of between 11 and 16 ageneses encountered in the permanent dentition, which correlate with a severe oligodontia phenotype.

A characteristic topographic distribution of congenitally missing teeth is encountered in HED, and this helps to determine the clinical and molecular diagnosis ${ }^{9}$. Coronal abnormalities of size and of morphology are typically observed in HED, consisting of cone shaped teeth and microdontia (Figs. 1-2).

b) The craniofacial phenotype described in the various forms of HED combine a large number of dys- morphic elements such as hypoplasia of the lower third of the face, maxillary hypoplasia, protruded or pseudoprotruded chin, facial concavity and a high forehead for example $8,13,21$ (Fig. 3). The causative mechanisms implicated in these skeletal abnormalities are multifactorial, and developmental and functional components apparently contribute to the craniofacial phenotype. EDA is a morphogenic factor that plays a role during embryogenesis of the mandible and of the facial bones, as well as in the mechanisms of differentiation and osteoblast proliferation ${ }^{4,8}$. Therefore, a mutation of the genes coding for EDA or its EDAR receptor, may affect the embryonic skeletal development of the facial bones. In addition, multiple congenitally missing teeth directly impact bone growth, that is clearly shown by the presence of correlations between the severity of oligodontia and the severity of the craniofacial phenotype in patients with $\mathrm{HED}^{13}$. 


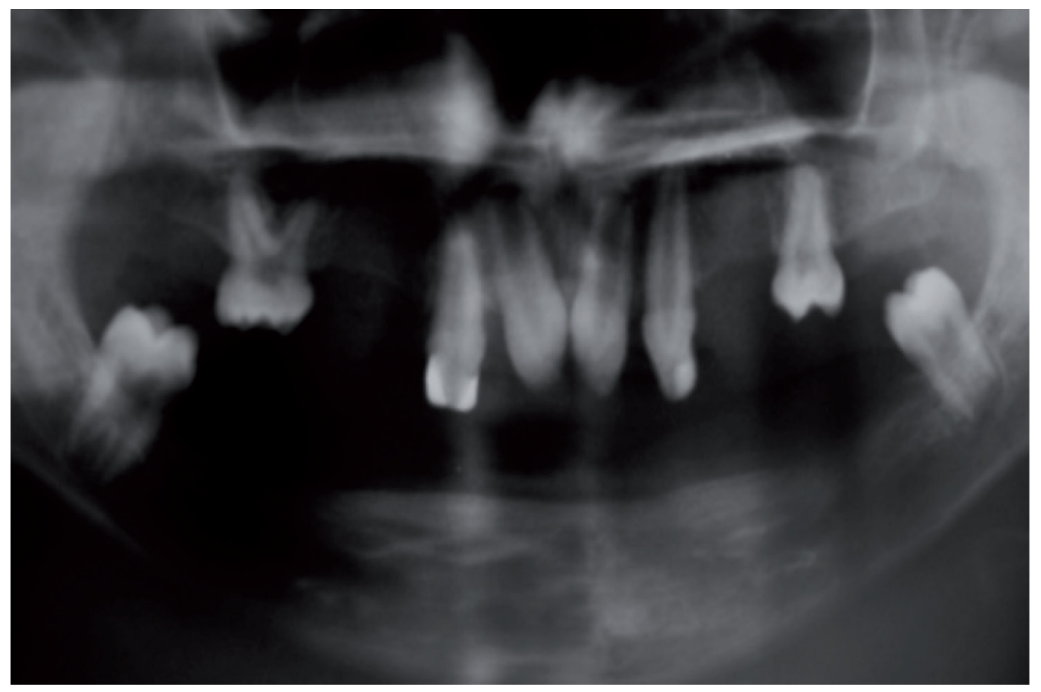

Figure 2

Characteristic radiological picture of hypohidrotic ectodermal dysplasia in the x-linked form of an 8 year old patient: severe oligodontia, with abnormal coronal morphology, dental misalignment, gaps.

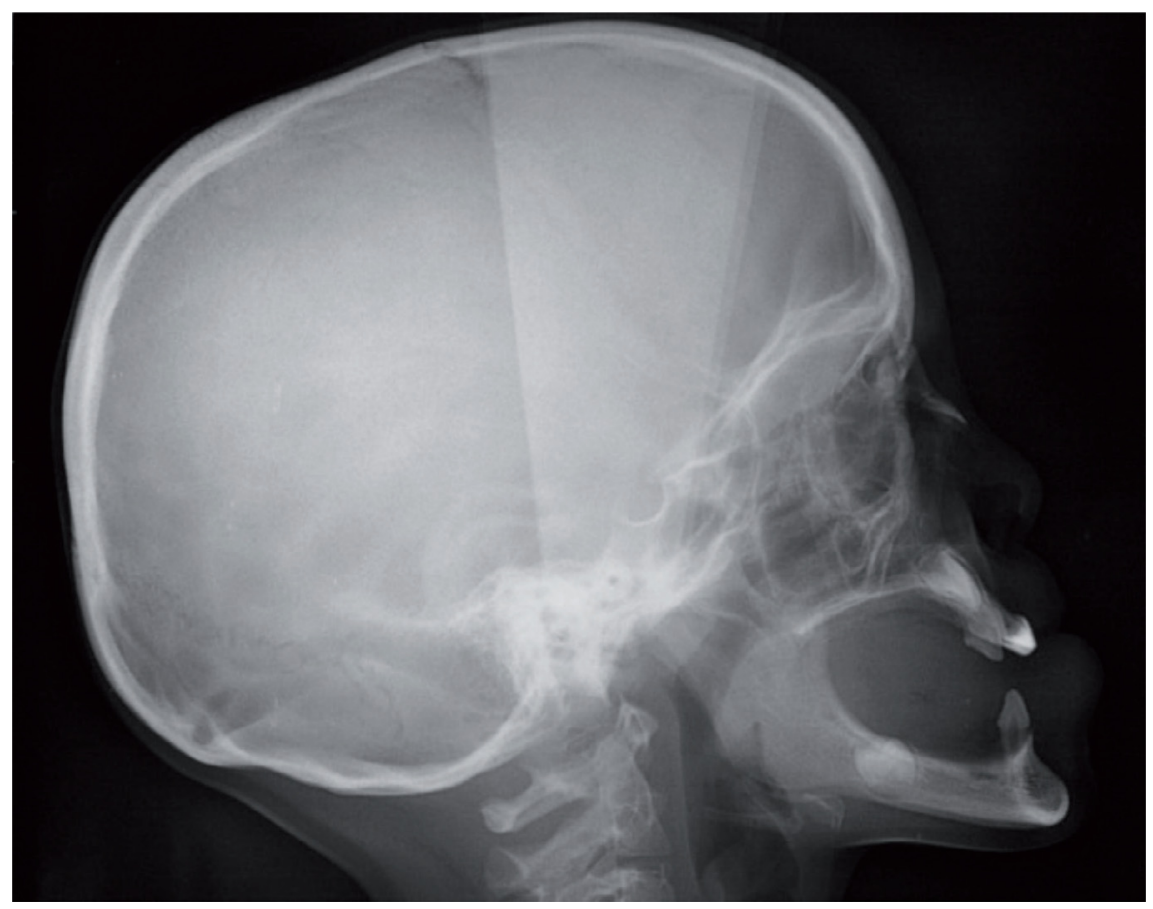

Figure 3

Cephalometric aspects of hypohidrotic ectodermal dysplasia in the x-linked form (5 year old patient with severe oligodontia). 
Oligodontia is the cause of many orofacial parafunctions and dysfunctions, especially masticatory ones, that contribute to the establishment of a dysmorphic and dysfunctional spiral and therefore of the observed phenotype. The various orofacial dysfunctions associated with HED have been the focus of clinical studies and evaluations using the Nordic scale of orofacial dysfunctions ${ }^{3}$. The tendency toward mandibular prognathism is ex- acerbated by a functional sliding forward during opening-closing movements and mastication associated with an absence of calibration between the opposing teeth in the anterior and posterior regions. It must also be noted that implant-prosthetic therapy does not create complete normalization of the cephalometric parameters and of growth in children and adolescents with HED, except for the ENA-ENP distance ${ }^{13}$.

\section{3 - MAKE UP OF THE MULTIDISCIPLINARY TEAM AND CENTRAL ROLE OF DENTO FACIAL ORTHOPEDICS (DFO)}

\section{a - Medical-surgical team}

A comprehensive multidisciplinary medical team must be assembled for treatment management of patients with HED. Specialists in medical genetics and pediatric dermatology take part in the initial diagnostic work-up, setting up biological and molecular examinations (looking for mutations), as well as genetic counseling. Pneumology, ophthalmology, gastroenterology, ENT and medical imaging are other important medical disciplines for the treatment management of these patients, given the various disorders and phenotype associated with HED. Maxillofacial specialists will intervene in particular for orthognathic surgery, pre-implantation bone grafts, even surgical repairs of labiopalatal clefts associated with some forms of HED with extra-ectodermal defects.

\section{b - Dental and surgical team}

Pediatric dentistry takes a role in the early treatment plan, particularly for the initial prosthetic treatment and prophylactic follow up for patients with HED. During this previous phase, coordination with specialists from maxillofacial imaging, implant and prosthetic surgery is required in the course of implant and prosthetic therapy.

\section{c - DFO}

Dentofacial orthopedics plays a central and primary role in the treatment management of patients with HED, beginning from the very first phases of therapy. In fact, interceptive and early orthopedic treatments can be planned, in cases of severe hypoplasia of the maxilla. Screening and early treatment of the parafunctions and dysfunctions are also important. Orthodontic procedures are performed during the pre-prosthetic, pre-surgical and pre or post-implant phase. 


\section{4 - ROLE OF DFO IN EARLY DIAGNOSIS}

\section{a - Analysis of skeletal maturity}

An analysis of the growth potential of different facial structures in patients with HED has shown decreased growth between the ages of 7 and 10, whereas it increased between the ages of 11 and 14. The growth spurt during puberty occurs approximately 2 years later and therefore must be taken into consideration for the orthopedic treatment plan, which may then be delayed accordingly ${ }^{10}$.

\section{b - Cephalometric features of patients with HED}

Cephalometric features of patients who are carriers of the various forms of HED have been the focus of a number of studies ${ }^{13,17}$. Globally, a growth deficiency in the middle third of the face has been observed. Skeletal and craniofacial manifestations are primarily maxillary hypoplasia with broad face (fig. 3). Based on cephalometric analysis, this is manifested by a reduction of the SNA angle, along with the ENA-ENP distance ${ }^{13}$. A reduction of the SN-ENAENP angle, correlating to a change in the maxillary orientation, was evident in patients with $\mathrm{HED}^{13}$. Regarding transverse dimensions, a narrow maxilla is also present. Measurement analysis of study models has shown however that the depth of the palate was respected. A protruded position of the mandible, associated with a decrease in the length of mandibular body is also typical and correlates to an increase of the SNPg angle according to cephalometric analysis, as well as to a decrease in the Go-Pg distance $^{13}$. An increase in the ENA/ ENP-Me/Go angle is also present in patients with HED, as is a reduction in ramus height that correlates to a decrease in the Go-Ar distance ${ }^{13}$. These different elements contribute to mandibular dysplasia tending toward skeletal Class III and protruded chin associated with maxillary brachygnathia $^{17}$ (Fig. 3). Statistical correlations have been made between the number of maxillary missing teeth and the severity of the craniofacial morphological abnormalities ${ }^{13}$.

In the vertical dimension, the repercussions of oligodontia are widespread and are the cause of reductions in total facial heights, both inferior and superior, manifested by a decrease in N-ME, ENA-Me and $N$-ENA distances ${ }^{13}$. The base of the skull appears just as affected in this HED; a decrease in the anterior region of the base of the skull, an increase in the posterior distance of the base of the skull (S-Ba), as well as a decrease in the basicranial angle has already been described ${ }^{8,13}$. A significant variation in the location of the ethmoid bone in relation to posterior nasal spine, correlating with the cephalometric parameters to an increase in the Er-ENP distance, has also been described in a group of patients with $\mathrm{HED}^{13}$.

In addition, a decrease in the distances between the hyoid bone and $\mathrm{C} 3$, and the hyoid bone and the mandibular plane, was also identified in a group of 10 HED patients. Significant cephalometric variations have been demonstrated in the soft tissues 
showing significant decreases in vertical length of the airway, in lingual length and an increase in the length of the soft palate. The facial profile is additionally characterized by a short nose, protruded lips, a decrease in convexity and facial heights ${ }^{17}$. Interestingly, the facial profile of vectoral heterozygotic (heterozygous carrier) females with the EDA mutation is also affected, presenting with protruding lips ${ }^{17}$.

\section{5 - ORTHOPEDIC PROCEDURES FOR PATIENTS WITH HED}

\section{a - Treatment plan for maxillary hypoplasia and mandibular prognathism}

Dentoalveolar and basal bone dysmorphisms associated with HED must be diagnosed and interceptive treatment management initiated as soon as possible. An early orthopedic procedure for maxillary bone growth can turn out to be beneficial for these patients with reduced vertical height, retrognathic jaws as well as a narrow chin, signs frequently found in $\mathrm{HED}^{12}$. The principal therapeutic objectives are transverse expansion of the maxilla, associated with anterior repositioning of the maxillary and a stimulation of sagittal expansion of the maxilla.

For the transverse orthopedic treatment plan, early installment of a quadhelix or a removable appliance with an expansion screw is indicated. Another solution can be the use of a central activator along with a connected pediatric prosthetic appliance, that makes it possible to combine prosthetic and orthopedic treatments $^{12}$.

For older patients assuming there is sufficient anchorage, a quadhelixcan be used for transverse maxillary expansion. This type of device is only feasible when phenotypes present moderate oligodontia with teeth in the posterior sectors that can be used for anchorage.

Treatment for a retrognathic maxilla, in turn, is based on anterior orthopedic positioning of the maxilla by using a facial mask. Stimulation of maxillary expansion in the sagittal dimension is intended to improve the facial profile that is characterized mainly by hypoplasia of the middle third of the face. The first stage of treatment with a facial mask consists in raising the incisal overbite. The use of a facial mask combined with an expander makes it possible to stimulate maxillary growth in the vertical, transverse and antero-posterior dimensions ${ }^{19}$.

Regarding a mandibular protrusion, early orthopedic restriction of mandibular growth can be planned for the skeletal Class III. However, in the majority of cases, it is not really a skeletal Class III but pseudo mandibular prognathism due to maxillary hypoplasia. Therefore, orthopedic repositioning of the maxillary, along with stimulation of antero-posterior growth will help to normalize sagittal skeletal relationships.

In the cases of confirmed skeletal Class III that are treated late, 
orthognathic surgery with a bilateral sagittal osteotomy of the rami combined with orthodontic treatment appears to be the best therapeutic option $^{18}$.

\section{6 - ORTHODONTIC THERAPIES FOR PATIENTS WITH HED}

\section{a - Pre-implant orthodontic treatments}

Orthodontic space opening, in order to get ideal implant placement, must be planned during the initial assessment. In fact, in cases of moderate oligodontia, orthodontic opening of certain spaces will make it possible to create a minimal space for the placement of an implant. Actually, a minimal space of $2 \mathrm{~mm}$ between an implant and a tooth must be respected. Besides measuring the inter-coronal distance when the edentulous space is bordered by teeth, the inter-radicular distance must also be assessed and possibly modified during orthodontic treatment, to avoid any implant-to-root proximity.

\section{b - Pre-prosthetic orthodontic treatments}

Pre-prosthetic orthodontic site preparation for opening space is an important step in the multi-disciplinary treatment plan for patients with HED (Figs. 4a-b). The therapeutic objective will be to create a balanced and harmonious appearance of the teeth on the arch, especially for patients who present moderate phenotypes of oligodontia that will benefit from implants and from prosthetic treatments with fixed implants and stabilized pros- thesis. Closing spaces created by congenitally missing teeth could incur the risk of having harmful consequences for the facial profile by exacerbating its concavity, and this explains why opening space is more appropriate for prosthetic restoration ${ }^{23}$. The treatment phase of post-orthodontic retention is essential at this stage in order to have long-term stability of the ideal positions of existing teeth by implant prosthetic restoration ${ }^{5}$.

\section{Role of temporary skeletal anchorage}

Cortical miniscrews used for skeletal anchorage are a useful and pertinent orthodontic tool, especially for patients with HED who present inadequate anchorage ${ }^{7,22}$. In fact, an evaluation of anchorage is an important parameter in orthodontic therapy, and this explains why different skeletal anchorage systems have been developed, particularly orthodontic mini-implants, palatal implants, mini-screws and plates ${ }^{15}$. These various appliances are classified based on their diameter, their length and whether or not there is osteointegration ${ }^{15}$.

Histological and clinical studies have shown that even with reduced lengths and diameters, these miniscrews make it possible to achieve a stable and adequate skeletal anchorage in relation to the 

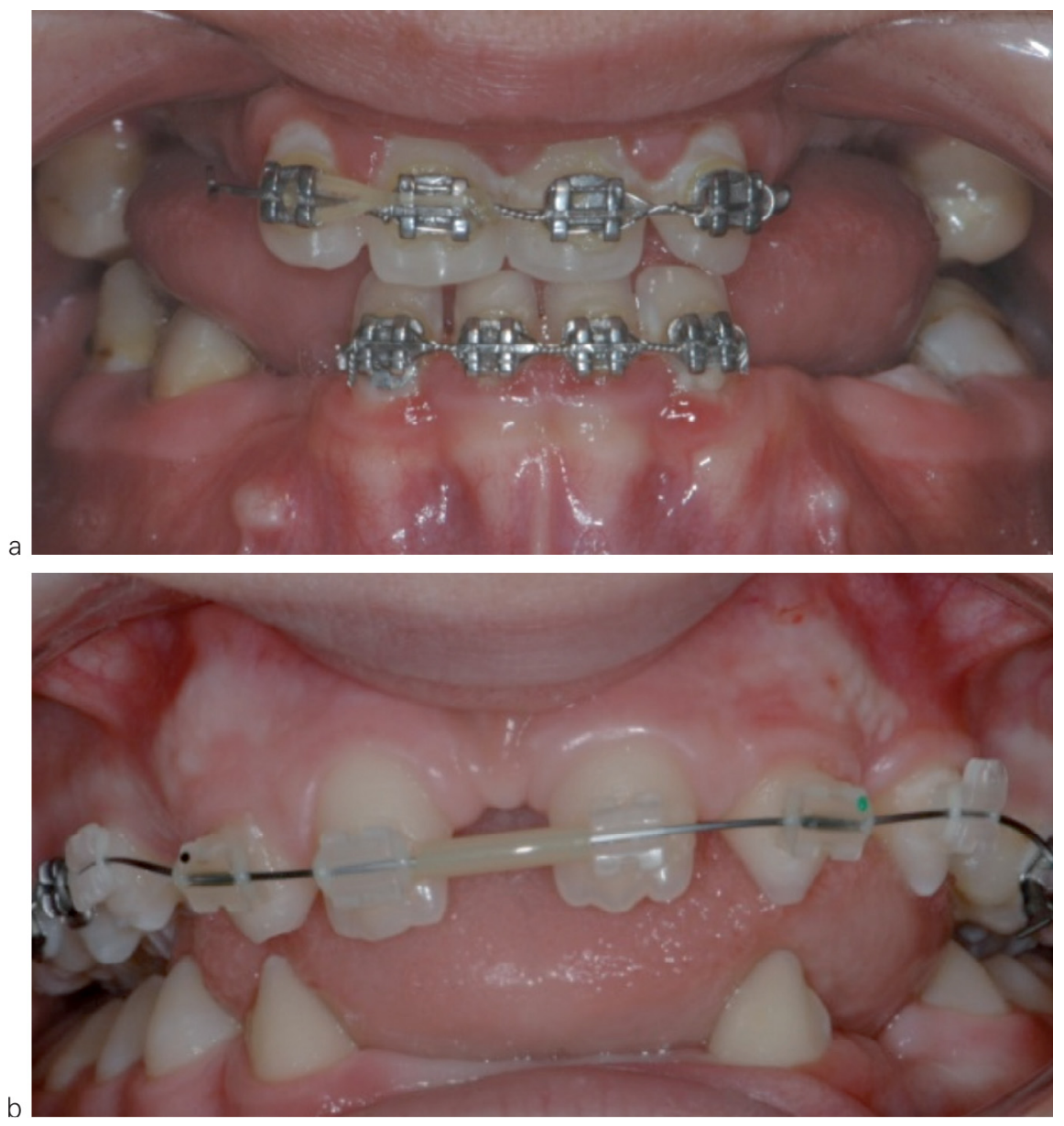

Figures $4 a-b$

Pre-prosthetic orthodontic treatments for two patients presenting moderate oligodontia associated with a HED: orthodontic management of space opening before implant prosthetic restoration.

orthodontic forces, including a shortened period of time for bone healing and a clinical rate of success better than $80 \%$. These success rates are slightly lower for intrusion of maxillary molars which could be explained by the quality and density of cortical bone. Therefore, installing screws on the zygomatic bone or on the hard palate is preferable since the palatal cortex is thicker and the quantity of attached gingiva is adequate $^{14}$. These appliances make it possible to perform orthodontic treatments that until now would have been impossible due to the lack of anchorage. In addition, they can also shorten the time of treatment.

Orthodontic mini-screws appear to be the best alternative because the surgery is atraumatic, anatomical restrictions are reduced, post-operative effects are favorable and make it 
possible to have skeletal anchorage comparable to mini-implants. Orthodontic treatment for oligodontia and malocclusion associated with HED can take full advantage of these orthodontic mini-screws, particularly the ones placed on the mandibular ramus $^{14}$. Mini-screws are also highly recommended for orthodontic intrusions of teeth with no opposing dentition, in cases of moderate or severe oligodontia with insufficient anchorage. For severe dysmorphisms, miniscrewassisted nonsurgical rapid transverse maxillary expansion can be followed

\section{7 - ORTHOGNATHIC SURGERY}

\section{a - Pre-surgical orthodontic preparation of the arches}

Orthodontic preparation of the arches is essential before single or double jaw orthognathic surgery. This orthodontic phase will make it possible to achieve occlusal references that will be used during the surgical movement of the basal bones and is generally done with multi-bracket attachment appliances. The main objectives of this pre-surgical stage are an alignment of the teeth on the arches, leveling of the Curve of Spee and treatment for alveolar protrusion and retrusion. Preoperative preparation of the arch wires used for retention and fabrication of the surgical stent for dental intercuspation are also necessary during this phase.

\section{b - Different types of surgery}

Orthognathic surgery such as Lefort 1 maxillary advancement surgery is by orthognathic surgery to correct the sagittal relationships ${ }^{16}$.

\section{c - Post implant orthodontic treatment}

Osteointegrated implants can be used as maximal anchorage during orthodontic treatment for post implant care in order to coordinate the occlusal relationships of the molars, to correct molar rotations and to close residual gaps ${ }^{24}$. A correction of possible implant-root proximities may also be indicated.

indicated in cases of severely retruded chin with abnormal shortness of the lower jaw ${ }^{1,20}$. Double jaw orthognathic surgery combining Lefort 1 osteotomy and correction of the mandibular prognathism by sagittal split according to Epker, can also be performed when dealing with severe phenotypes ${ }^{20}$.

Different surgical sequences are conceivable, combining orthognathic surgery, bone graft and implant surgery. Therefore, the first surgical procedure may involve a Lefort 1 osteotomy with maxillary sinus floor lift elevation and autologous bone grafts applied laterally to the cortical bone. After a period of bone healing and engraftment, with a healing time which should be preferably lengthened when dealing with syndromes, the second surgical procedure is the placement of the osteointegrated implants ${ }^{1}$. A combination of single or double jaw orthognathic surgery and placement 
of orthodontic miniscrews may also be indicated, as Kuroda et al. ${ }^{14}$ in particular have described, by performing a Lefort 1 osteotomy, a sagittal split (Epker) and placement of miniscrews on the anterior area of the ascending ramus.

\section{c - Post-surgical orthodontic finishing}

A multi-bracket attachment orthodontic treatment for orthodontic finishing is generally indicated after surgical correction of the skeletal relationships. It will mainly involve closing residual spaces and represents the last therapeutic stage of treatment.

The growth of these patients also includes certain specificities, a delay in the growth spurt during puberty that must be taken into account from an orthopedic perspective.

Temporary skeletal anchorage with miniscrews or plates can also be used for phenotypes of severe oligodontia characterized by inadequate anchorage. Single or double jaw orthognathic surgery, with or without pre-implant surgery, is indicated in cases of severe dysmorphism. should benefit from early diagnosis and early orthopedic management.

\section{REFERENCES FOR CONSULTATION}

1. Bayat M, Khobyari MM, Dalband M, Momen-Heravi F. Full mouth implant rehabilitation of a patient with ectodermal dysplasia after orthognathic surgery, sinus and ridge augmentation: a clinical report. J Adv Prosthodont 2011;3:96-100.

2. Bergendal B. Children with ectodermal dysplasia need early treatment. Spec Care Dentist 2002;22(6):212-213.

3. Bergendal B, McAllister A, Stecksén-Blicks C. Orofacial dysfunction in ectodermal dysplasias measured using the Nordic Orofacial Test-Screening protocol. Acta Odontol Scand 2009;67:377-381.

4. Bornert F, Choquet P, Gros Cl, Aubertin G, Perrin-Schmitt F, Clauss F, Lesot H, Constantinesco A, Schmittbuhl M Subtle Morphological Changes in the Mandible of Tabby Mice Revealed by Micro-CT Imaging and Elliptical Fourier Quantification. Front Physiol 2011;2:15. doi: 10.3389/fphys.2011.00015.

5. Borzabadi-Farahani A. Orthodontic considerations in restorative management of hypodontia patients with endosseous implants. J Oral Implantol 2012;38:779-791. 
6. Chassaing N, Bourthoumieu S, Cossee M, Calvas P, Vincent MC. Mutations in EDAR account for one-quarter of non-ED1-related hypohidrotic ectodermal dysplasia. Hum Mutat 2006;27:255-259.

7. Chillès JG, Riemenschneider-Chillès $S$. Use of short cortical mini-screws in orthodontics. Orthod Fr 2011;82:253-268.

8. Clauss F, Manière MC, Obry F, Waltmann E, Hadj-Rabia S, Bodemer C, Alembik Y, Lesot $\mathrm{H}$, Schmittbuhl M. Dento-craniofacial phenotypes and underlying molecular mechanisms in hypohidrotic ectodermal dysplasia (HED): a review. J Dent Res 2008;87(12):1089-1099.

9. Clauss F, Chassaing N, Smahi A, Vincent MC, Calvas P, Molla M, Lesot H, Alembik Y, Hadj-Rabia S, Bodemer C, Manière MC, Schmittbuhl M. X-linked and autosomal recessive Hypohidrotic Ectodermal Dysplasia: genotypic-dental phenotypic findings. Clin Genet 2010;78:257-266.

10. Dellavia C, Catti F, Sforza C, Grandi G, Ferrario VF. Non-invasive longitudinal assessment of facial growth in children and adolescents with hypohidrotic ectodermal dysplasia. Eur J Oral Sci 2008;116:305-311.

11. Gündüz Arslan S, Devecioglu Kama J, Ozer T, Yavuz I. Craniofacial and upper airway cephalometrics in hypohidrotic ectodermal dysplasia. Dentomaxillofac Radiol 2007;36:478-483.

12. Ioannidou-Marathiotou I, Kotsiomiti E, Gioka C. The contribution of orthodontics to the prosthodontic treatment of ectodermal dysplasia: a long-term clinical report. J Am Dent Assoc 2010;141:1340- 1345.

13. Johnson EL, Roberts MW, Guckes AD, Bailey LJ, Phillips CL, Wright JT. Analysis of craniofacial development in children with hypohidrotic ectodermal dysplasia. Am J Med Genet 2002;112:327- 334.

14. Kuroda S, Sugawara Y, Yamashita K, Mano T, Takano-Yamamoto T. Skeletal Class III oligodontia patient treated with titanium screw anchorage and orthognathic surgery. Am J Orthod Dentofacial Orthop 2005;127:730-738.

15. Labanauskaite B, Jankauskas G, Vasiliauskas A, Haffar N. Implants for orthodontic anchorage. Meta-analysis. Stomatologija 2005;7:128-132.

16. Lee KJ, Park YC, Park JY, Hwang WS. Miniscrew-assisted nonsurgical palatal expansion before orthognathic surgery for a patient with severe mandibular prognathism. Am J Orthod Dentofacial Orthop 2010;137:830-839.

17. Lexner MO, Bardow A, Bjorn-Jorgensen J, Hertz JM, Almer L, Kreiborg S. Anthropometric and cephalometric measurements in X-linked hypohidrotic ectodermal dysplasia. Orthod Craniofac Res 2007;10:203-215.

18. Ravi MS, Shetty NK, Prasad RB. Orthodontics-surgical combination therapy for Class III skeletal malocclusion. Contemp Clin Dent 2012;3:78-82. doi: 10.4103/0976237X.94552.

19. Raymond J.-L. Traitement orthopédique des malocclusions de classe III. Empresa 160 p. 2011.

20. Ritto FG, Medeiros PJ, de Oliveira Mussel RL, de-Sá-Silva E. Rehabilitation of an adolescent with ectodermal dysplasia. Two-stage orthognathic, graft, and implant surgery: case report. Implant Dent 2009;18:311-315.

21. Ruhin B, Martinot V, Lafforgue P, Catteau B, Manouvrier-Hanu S, Ferri J. Pure ectodermal dysplasia: retrospective study of 16 cases and literature review. Cleft Palate Craniofac J 2001;38:504-518.

22. Stanford N. Mini-screws success rates sufficient for orthodontic treatment. Evid Based Dent 2011;12:19. 
23. Valle $A L$, Lorenzoni $F C$, Martins $L M$, Valle CV, Henriques JF, Almeida AL, Pegoraro LF. A multidisciplinary approach for the management of hypodontia: case report. J Appl Oral Sci 2011;19:544-548.

24. Van Sickels JE, Raybould TP, Hicks EP. Interdisciplinary management of patients with ectodermal dysplasia. J Oral Implantol 2010;36:239-245.

25. Worsaae N, Jensen BN, Holm B, Holsko J. Treatment of severe hypodontia-oligodontia-an interdisciplinary concept. Int J Oral Maxillofac Surg 2007;36:473-480.

26. Yavuz I, Baskan Z, Ulku R, Dulgergil TC, Dari O, Ece A, Yavuz Y, Dari KO. Ectodermal dysplasia: Retrospective study of fifteen cases. Arch Med Res 2006;37:403-409. 